\title{
Interplay of Dzyaloshinsky-Moriya and Dipole-Dipole Interactions and Their Joint Effects upon Vortical Structures on Nanodisks
}

\author{
Zhaosen $\mathrm{Liu}^{a 1}$, Orion $\mathrm{Ciftja}^{b}$, Hou $\operatorname{Ian}^{c 2}$ \\ ${ }^{a}$ Department of Applied Physics, University of Information Science and Technology, \\ Nanjing 210044, China \\ ${ }^{b}$ Department of Physics, Prairie View A\&M University, Prairie View, TX 77446, USA \\ ${ }^{c}$ Institute of Applied Physics and Materials Engineering, FST, University of Macau, China
}

\begin{abstract}
In transition metal oxides, magnetic dipole-dipole (DD) and chiral DzyaloshinskyMoriya (DM) interactions between nearest neighboring spins are comparable in magnitude. In particular, the effects of the DD interaction on the physical properties of magnetic nanosystems cannot be simply neglected due to its long-range character. For these reasons, we employed here a new quantum simulation approach in order to investigate the interplay of these two interactions and study their combined effects upon the magnetic vortical structures of monolayer nanodisks. Consequently, we found out from our computational results that, in the presence of Heisenberg exchange interaction, a sufficiently strong DD interaction is also able to induce a single magnetic vortex on a small nanodisk; a strong DM interaction usually gives rise to a multi-domain structure which evolves with changing temperature; In this circumstance, if a weak DD interaction is further considered, the multi-domains merge to form a single vortex in the whole magnetic phase. Moreover, if only the Heisenberg exchange and chiral DM interactions are considered in simulations, our results from calculations with different spin values show that the transition temperature $T_{M}$ is simply proportional to $S(S+1)$; if the temperature is scaled with $T_{M}$, and the calculated magnetizations are divided by the spin value $S$, their curves exhibit very similar features in the whole temperature region below $T_{M}$.
\end{abstract}

Keywords: Dzyaloshinsky-Moriya Interaction, Dipole-Dipole Interaction,

\footnotetext{
${ }^{1}$ Email: liuzhsnj@yahoo.com

${ }^{2}$ Email: houian@umac.mo
} 
Magnetic Vortices, Quantum Simulation Model

\section{Introduction}

In 1989, Bogdanov and Yablonskii speculated theoretically [1] that skyrmion$\mathrm{s}$, which were originally introduced in particle physics to describe the localized, particle-like topological solitons [2], can exist in magnets when the chiral DzyaloshinskyMoriya (DM) interaction is present $[3,4,5]$. This prediction was later confirmed by experimental findings that magnetic skyrmions can be generated by the DM interaction in helical magnets, such as $\mathrm{MnSi}$ and $\mathrm{Fe}_{1-x} \mathrm{Co}_{x} \mathrm{Si}[6,7,8]$. The results suggest that this interaction favors canted spin configurations $[6,7,8,9,10,11,12,13,14$, 15, 16]. Most skyrmions found in helimagnets were induced by an external magnetic field at low temperatures [6, 7, 8, 17]. However, Yu et al. [7] obtained a skyrmion lattice in FeGe with a high transition temperature around $280 \mathrm{~K}$ by applying an external magnetic field, as well.

The diameters of the skyrmion structures formed in a Fe monolayer on an $\operatorname{Ir}(111)$ surface are in the order of a few atomic spacings [10]. They can be easily manipulated by spin currents of only about $10^{6} \mathrm{Am}^{-2}[18,19]$, which is a factor of $10^{5}$ to $10^{6}$ weaker than those required to drive magnetic domain walls [20]. These unique properties provide a possibility to fabricate novel high density, power-efficient spintronic storage and logic devices [17, 21], for example, a racetrack-like memory [22] that uses skyrmions instead of magnetic domains [21, 23].

Both magnetic skyrmions and vortices, are usually induced by the DM interaction. Thus, they are closely related and have been mainly studied numerically with Monte Carlo and micromagnetic methods. Within the framework of both these methods, the spins and magnetic moments in the sample under consideration are treated as classical vectors with fixed lengths. In order to go beyond a classical treatment, we have developed a quantum simulation approach in recent years $[24,25,26,27]$. In this new method, all spins are taken as quantum operators, and all physical quantities are calculated strictly with quantum theory. Thus the computational code can quickly converges to the correct equilibrium states spontaneously at all temperatures.

Recently, we employed this quantum simulation approach to investigate the magnetic and thermodynamic properties of both ferromagnetic (FM) and antiferromagnetic (AFM) monolayer nanodisks in the presence of a chiral DM interaction[28, 29]. In the first case, we found that the chirality of the FM vortices formed on a small nanodisk is solely determined by the sign of DM interaction parameter, and the 
applied magnetic field is able to stabilize the single vortical structure $[6,7,8,17]$. In the second case, we observed that the magnetic moments not only form in-plane AFM vortices, but also order antiferromagnetically in the perpendicular direction. In the both cases, the calculated average distances between two neighboring vortices agree well with a grid theory in the low temperature region [30].

On the other hand, in transition metal oxides, the DM interaction is of the order of $0.1 \mathrm{meV}$ between a pair of nearest neighbouring spins, while that for the dipoledipole (DD) interaction is around $\mu_{0} \mu_{B}^{2} / 4 \pi a^{3} \sim 0.1 \mathrm{meV}$, which is comparable to the DM interaction [31]. Moreover, the dipolar interaction is a long-range interaction, so it plays an important role in the formation of long-range ordered spin structures. In particular, its co-existence with the Heisenberg exchange can also generate magnetic vortices on nanodisks $[32,33,34,35,36,37]$. When this long-range interaction is sufficiently strong, the continuity of the effective magnetic field along the boundary of a two dimensional (2D) nanodot is able to force the magnetic moments to align tangent to the border [38, 39, 40], thus a vortex appears on the disk-plane [41].

So far, a great amount of works in this respect have been undertaken over the years, such as Monte Carlo simulations [41, 42, 43, 44], micromagnetic simulations [45], computations for dynamics of vortex [46], theoretical studies on the effect of the DD interaction [47] and experimental studies [48, 49, 50]. Vortices and anti-vortices were observed experimentally [49] in 2D cobalt micro-dots; layers of magnetic dots on a superconducting film, which shows transport properties attributed to magnetic vortices, were also investigated [50].

In the present work, we employed our new quantum simulation approach to investigate the effects of the DM and DD interactions upon the vortical structures on a circular magnetic monolayer nanodisk with the square unit cell structure consisting of $S=3 / 2$ spins. We found that, in presence of Heisenberg exchange interaction, a sufficiently strong DD interaction is also able to induce a single stable magnetic vortex on a small nanodisk; whereas a strong DM interaction usually gives rise to temperature-dependent multi-vortical structures - however, few people have paid attention to, or systematically studied the dynamic character of such phenomenon; and when a strong DM and a weak DD interactions are both involved, the above multi-domain structures merge to form a single vortex which prevails in the whole magnetic phase. Moreover, if only Heisenberg exchange and chiral DM interactions are considered, our results obtained for different spin values indicate that the transition temperature $T_{M}$ is simply proportional to $S(S+1)$; and if the temperature is scaled with $T_{M}$, the calculated $\left\langle S_{x}\right\rangle,\left\langle S_{y}\right\rangle$ and $\left\langle S_{z}\right\rangle$ are divided by the spin value $S$, their curves only differ slightly in the whole magnetic phase. 


\section{Quantum Model and Computational Algorithm}

The Hamiltonian of the nanosystems that we consider here can be expressed as $[30,51,52]$

$$
\begin{gathered}
\mathcal{H}=-\frac{1}{2} \sum_{i, j \neq i}\left[\mathcal{J}_{i j} \vec{S}_{i} \cdot \vec{S}_{j}-D_{i j} \vec{r}_{i j} \cdot\left(\vec{S}_{i} \times \vec{S}_{j}\right)\right] \\
-\frac{d}{2} \sum_{i, j \neq i}\left[\frac{3\left(\vec{s}_{i} \cdot \vec{r}_{j j}\right)\left(\vec{s}_{j} \cdot \vec{r}_{i j}\right)}{r_{i j}^{5}}-\frac{\vec{s}_{i} \cdot \vec{s}_{j}}{r_{i j}^{3}}\right]
\end{gathered}
$$

where the first and second terms represent the Heisenberg exchange and DM interactions between every pair of neighboring spins with the strengths of $\mathcal{J}_{i j}$ and $D_{i j}$, respectively. The last term denotes the DD interaction, where $\vec{r}_{i j}$ is the vector from the $i$ th to the $j$ th sites, and $d$ is the dipolar interaction strength. The last sum is taken over all spins within a circle of a cut-off radius $r_{c}$. To a satisfactory accuracy, $r_{c}$ was set to $15 a$ in all our simulations.

The nanodisks under consideration are assumed to be composed of $S=3 / 2$ spins. In light of quantum theory, the matrices of the spin components in a cartesian system are given by

$$
\begin{aligned}
& S_{x}=\frac{1}{2}\left(\begin{array}{cccc}
0 & \sqrt{3} & 0 & 0 \\
\sqrt{3} & 0 & 2 & 0 \\
0 & 2 & 0 & \sqrt{3} \\
0 & 0 & \sqrt{3} & 0
\end{array}\right), S_{y}=\frac{1}{2 i}\left(\begin{array}{cccc}
0 & \sqrt{3} & 0 & 0 \\
-\sqrt{3} & 0 & 2 & 0 \\
0 & -2 & 0 & \sqrt{3} \\
0 & 0 & -\sqrt{3} & 0
\end{array}\right), \\
& S_{z}=\frac{1}{2}\left(\begin{array}{cccc}
3 & 0 & 0 & 0 \\
0 & 1 & 0 & 0 \\
0 & 0 & -1 & 0 \\
0 & 0 & 0 & -3
\end{array}\right),
\end{aligned}
$$

respectively.

As described in our previously published papers [24, 25, 28, 29], all simulations done in recent years are started from a random magnetic configuration above the magnetic transition temperature $T_{M}$, then they are carried out stepwise down to very low temperatures. At a temperature below $T_{M}$, convergency is considered to be reached if the difference $\left(\left|\left\langle\vec{S}_{i}^{\prime}\right\rangle-\left\langle\vec{S}_{i}\right\rangle\right|\right) /\left|\left\langle\vec{S}_{i}\right\rangle\right|$ between two successive iterations for every spin is less than a very small given value $\tau_{0}$.

\section{Simulated Results}

In order to clearly display the spin configurations, we considered here a very small circular monolayer nanodisk with radius $R=10 a$, where $a$ is the side length of a square crystal unit cell. For convenience, only the interactions between the 
nearest neighboring spins are considered and these spins are coupled uniformly with strengths $\mathcal{J}_{i j}=\mathcal{J}=1 \mathrm{~K}$ and $D_{i j}=D$. Therefore, all parameters used in this work are scaled with both Boltzmann constant $k_{B}$ and the exchange strength $\mathcal{J}$.

\subsection{Temperature Dependence of Vortical Structure Driven by a Strong DM Inter- action}

Firstly, to investigate how the vortical structure evolves with the changing temperature under a strong DM interaction, DD interaction was neglected, and $D$ was assigned to a value of $0.3 \mathrm{~K}$ to perform simulations.
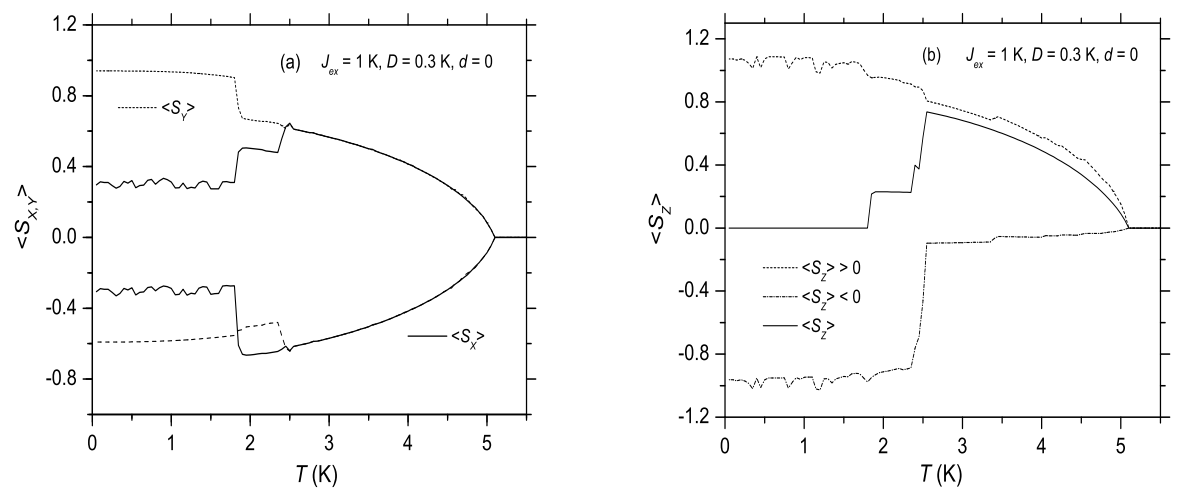

Figure 1. Calculated spontaneous (a) $\left\langle S_{x}\right\rangle,\left\langle S_{y}\right\rangle$, and (b) $\left\langle S_{z}\right\rangle$ for the monolayer FM nanodisk as a function of temperature. Here $R=10 \mathrm{a}, \mathcal{J}=1 \mathrm{~K}, D$ $=0.3 \mathrm{~K}$, and $d=0$, respectively.

Figure 1(a,b) displays our calculated thermally averaged $\left\langle S_{x}\right\rangle,\left\langle S_{y}\right\rangle$ and $\left\langle S_{z}\right\rangle$ for the nanodisk. The DM interaction has indeed induced an out-plane magnetic moment $\left\langle S_{z}\right\rangle$ below the transition temperature $T_{M} \approx 5.15 \mathrm{~K}[53,54,55,56]$, which is stronger than the other two components, $\left\langle S_{x}\right\rangle$ and $\left\langle S_{y}\right\rangle$, in the whole magnetic phase. The curves abruptly change at $T_{M 1,2,3,4} \approx 1.65,1.85,2.45$, and $2.50 \mathrm{~K}$, suggesting that a few spin configurations have been formed in the magnetic phase, as shown and described below. Above $T_{M 4}(\approx 2.50 \mathrm{~K})$, all the three components decrease monotonously as temperature increases, and below $T_{M 1}(\approx 1.65 \mathrm{~K})$, as expected, $\left\langle S_{z}\right\rangle$ increases gradually in magnitude, though not smoothly, as temperature decreases.

Under the strong DM interaction, ferromagnetic vortical structures are formed on the nanodisk, however, they are temperature dependent. Figure 2 displays the 
magnetic structures, recorded at four temperatures, projected onto the disk-plane (the $x y$-plane) and in the perpendicular $z$-direction.

As shown in Figure 2(a,b), a single vortex is observed in the disk-plane in the temperature region $T_{M 4} \leq T<T_{M}$. Inside the central circle of radius $r=8 a$, the $z$-components of all spins are upward, outside of this circle, the spins are downward. Therefore, the chirality of the single vortex is clearly right-handed since $D>0$. Obviously, the sizes of the marginal and central circular areas are affected by the disk size and temperature.

As temperature drops down, the spin configuration evolves. As shown in Figure $2(\mathrm{c})$, at $T=2.5 \mathrm{~K}$, an elongated vortex centered at the origin of the nanodisk appears, accompanied by other two initially developed vortices on its both sides. Figure 2(d) displays the $z$-projection of the spin structure. In the elongated area all spins are upward, others on its both sides are downward. Although the three vortices are deformed, their chiralities are still right-handed since $D$ is positive.

Down to $T=2 \mathrm{~K}$, the spin structure evolves into two oppositely curling vortices, with one better developed than the other, in the both sides of a wide strip, as shown in Figure 2(e,f).

The two vortices and the strip further evolve as the temperature decreases, they finally assume a spin pattern below $T=1.5 \mathrm{~K}$ as shown in Figure $2(\mathrm{~g}, \mathrm{~h})$. The spins in the strip are all upwards, the two oppositely curling vortices on its both sides are well symmetric about the $y$-axis.

By comparing Figure 2(e,g) with Figure 2(f,h) correspondingly, we find that the chiralities of the two vortices formed at the two temperatures are also right-handed.

To describe the self-organized multi-domain structures, a new quantity named DM length has been introduced and defined as $\zeta=\mathcal{J} / D$, where the distance between two nearest grids is considered to be one unit, and $\zeta$ represents the averaged distance between two neighboring magnetic domains [30]. We adopt this theory in this work by replacing the grid with a spin. For the case considered here, $\zeta=\mathcal{J} / D \approx 3.333$, and $2 R>\zeta$, so it is expected that more self-organized magnetic domains will appear in the low temperature region. The two vortex centers shown in Figure 2(g) are approximately $10 a$ apart and three self-organized domains are involved in between. Thus the averaged distance of two neighboring structures is about 3.333a, which agrees well with the gird theory [30].

To further describe the detailed spin configurations on the nanodisk, two other quantities are introduced and defined as

$$
A_{z}(r)=\frac{1}{N_{c}(r)} \sum_{i}\left|\left\langle S_{i z}\right\rangle\right|, \quad A_{x y}(r)=\frac{1}{N_{c}(r)} \sum_{i} \sqrt{\left\langle S_{i x}\right\rangle^{2}+\left\langle S_{i y}\right\rangle^{2}},
$$


for the out-of and in-plane components of the spins, respectively. Here $N_{c}(r)$ is the number of the spins on a circle of radius $r$ around the disk center. These two quantities were calculated at four different temperatures, and the results are displayed as the functions of $r$ in Figure $3(\mathrm{a}, \mathrm{b})$. When $T=5 \mathrm{~K}$ and $2.5 \mathrm{~K}, A_{z}\left(A_{x y}\right)$ has larger (weaker) magnitude in the circular core region around the disk center, but relatively weaker (larger) magnitude in the marginal area; two peaks appear at $r \approx 5 a$ and $8 a$ in the $A_{x y}$ curves at the two temperatures, respectively, as shown in Figure 3(a); and the two sets of the curves are considerably reduced as temperature is increased from $T=2.5 \mathrm{~K}$ to $5 \mathrm{~K}$ as expected. In the central region of the disk, $A_{x y}$ is considerably weaker than $A_{z}$. That is, therein the spin are mainly oriented ferromagnetically out of the plane, but slightly canted from the normal direction of the disk.

However, at both $T=2 \mathrm{~K}$ and $0.5 \mathrm{~K}$, as shown in Figure $3(\mathrm{~b}), A_{z}$ and $A_{x y}$ have exchanged their roles. For each $A_{z}$ curve, a deep valley appears in the central region, two round peaks emerge away from the nanodisk center; whereas for each $A_{x y}$ curve, a round peak appears around the disk center, two valleys emerge away from the center as well. The phenomena seem strange. However, as $T \geq 2.5 \mathrm{~K}$, a single vortex is formed and its center is the origin of the circular disk; but, as $T \leq$ $2 \mathrm{~K}$, the centers of the two vortices are at other places, whereas the disk center is now inside the strip which separates the two vortices.

By comparing Figure 3(a) to (b), one can conclude that, on average, no matter a single vortex or multi-vortices is/are formed on the nanodisk, $A_{z}$ is usually larger in the central region than in the marginal area of the vortex, whereas, $A_{x y}$ is weaker inside the central area than in the marginal region of the vortex. Towards the boundary of a vortex, $A_{z}$ decreases but $A_{x y}$ grows gradually both in magnitude on average. That is, in this process the spins are rotated by the effective magnetic field gradually towards the disk plane.

\subsection{Effects of Spin Value and General Scaling Rules}

We have previously done simulations for a nanodisk of the same size but consisting of $S=1$ spins using the same set of parameters [28]. The two sets of magnetization curves, as well as the spin configurations, show roughly similar features. However, the transition temperature has now been considerably increased, and the chirality of each deformed vortex caused by the strong DM interaction is here carefully studied. By comparing the two sets of calculated results, it is astonishing to find that the transition temperature $T_{M}$ is simply proportional to $S(S+1)$; and if the temperature is scaled with $T_{M}$, all the newly calculated $\left\langle S_{x}\right\rangle,\left\langle S_{y}\right\rangle$ and $\left\langle S_{z}\right\rangle$ are divided by the spin value $S$ (by $-S$ in this case), and the first two components 
are exchanged, thus, the re-plotted curves differ only slightly from those obtained before [28] in the whole low temperature range. To check these rules, we performed simulations for such a disk consisting of $S=5 / 2$ spins, and consequently noticed that these rules still hold true.

\subsection{Magnetic Vortex Induced by DD Interaction}

On the other hand, in the presence of Heisenberg exchange, a reasonably strong DD interaction is able to induce vortical structure on 2D nanodisks [32, 33, 34, 35, $36,37,41]$. To test this idea, we carried out simulations with the new quantum approach by excluding the DM interaction, but assigning values of $1 \mathrm{~K}$ and $0.2 \mathrm{~K}$ to $\mathcal{J}$ and $d$, respectively.

The calculated $\left\langle S_{x}\right\rangle,\left\langle S_{y}\right\rangle$ and $\left\langle S_{z}\right\rangle$ are displayed in Figures 4(a) and (b) respectively. Both the $x, y$ components decrease gradually and monotonously with rising temperature, though they fluctuate slightly especially above $3.5 \mathrm{~K}$. These two curves have almost the same shape, but are shifted vertically with respect to each other in the magnetic phase. In contrast, the upward and downward components of $\left\langle S_{z}\right\rangle$, as well as their total average, are six to seven orders weaker than $\left\langle S_{x}\right\rangle$ and $\left\langle S_{y}\right\rangle$ in magnitude, thus can be neglected.

The DD interaction expressed in Eq.(1) can be divided into two parts: the first part tends to rotate the spins to the direction of $\vec{r}_{i j}$ that is completely in the monolayer disk, whereas the second one attempts to align each pair of spins antiferromagnetically. Owing to its long-range character, several hundreds of spins are involved in this interaction. Since all spins are on the monlayer disk, the DD interaction will force the magnetic moments rotate towards the $x y$ plane, so we observed larger $\left\langle S_{x}\right\rangle$ and $\left\langle S_{y}\right\rangle$, but negligible $\left\langle S_{z}\right\rangle$.

When the magnetic moments in the border of a nanodisk are all aligned tangent to the border, both the Heisenberg exchange energy and the second part of the dipolar energy are minimized. Meanwhile, the second part of the DD interaction is also minimized between those spins at the opposite sites in relative to the nanodisk center. As a result, a single magnetic vortex can be formed as shown in Figure 5(a).

For the reason just explained, only $A_{x y}(r)$ is displayed in Figure $5(\mathrm{~b})$. There, we find that in low temperature region, for example at $T=0.6 \mathrm{~K}, A_{x y}$ is almost a constant with a saturated value $S=3 / 2$. However, in high temperature region, for instance at $T=3 \mathrm{~K}$ or $5 \mathrm{~K}, A_{x y}$ shows features similar to those displayed in Figure 3 : for each $A_{x y}$ curve, a deep valley appears in the central range, two peaks, but quite wider now, emerge around $r=5 a$. Since $S_{z}$ is negligible in the whole temperature range, it is meaningless to talk about the chirality of this kind of vortex induced by the DD interaction. 


\subsection{Interplay of DM and DD Interactions and Their Joint Effects on Vortical Struc- ture}

Based on the above findings, we naturally wonder how the spin configurations will look like and will evolve with changing temperature if the DM and DD interactions are both involved. To find the answer, we did additional simulations by setting $\mathcal{J}$, $D$ and $d$ to $1 \mathrm{~K}, 0.3 \mathrm{~K}$ and $0.01 \mathrm{~K}$, respectively.

The DD interaction is now considerably weak. However, the multi-domain structure generated by the DM interaction, as shown in Figure 2, has been completely overcomed by this weak long-range interaction, the temperature-dependent multidomains observed there are now replaced by a single vortex which is stable in the whole magnetic phase, as shown in Figure 6(a). Since $D$ is positive, the chirality of the vortex is still right-handed in the circular core region of the nanodisk, where the $z$ components of the spins are all downward as seen in Figure 6(b). However, in the marginal area, the $z$ components order in the opposite direction, and this area shrinks as temperature falls down.

The weak DD interaction of this strength cannot induce a vortical structure by itself [41]. However, the single vortex induced by the DM interaction just below the transition temperature, as shown in Figure 2(a), is now reinforced by the DD interaction, since it favors the same spin structure as well, so that the single vortex is able to persist down to very low temperatures.

Correspondingly, the step-like shapes of the magnetization curves observed in Figure 1 are now replaced by gradually changing plots as shown in Figure 7, suggesting that the single-vortex structure prevails in the whole magnetic phase. In comparison with the curves displayed in Figure 1, on average, $\left\langle S_{x}\right\rangle$ and $\left\langle S_{y}\right\rangle$ have now been enhanced, whereas $\left\langle S_{z}\right\rangle$ has been reduced, all by the DD interaction.

The calculated $A_{z}$ and $A_{x y}$ curves exhibit very similar features to those displayed in Figure 3. $A_{z}$ has its maximum in the core region; as $r$ increases, it falls gradually firstly, then increases. $A_{x y}$ has its maximum elsewhere, and it is considerably weak in the central region. Thus, in the circular region around the center, the spins order mainly normal to the disk plane. Away from the center, the $z$ component decreases, whereas the $x, y$ components increase firstly, then falls down. Then we can find a wide circular ring, where the spins align mainly in the disk plane. As temperature decreases, the ring moves outwards, and the in-plane component of the vortex is considerably enhanced. These curves are not shown here to avoid repetition. 


\section{Conclusions and Discussion}

We have employed a new quantum simulation approach to investigate the effects of the DM and DD interactions, when they are present separately or co-exist, upon the macroscopic physical properties and microscopic spin structures of monolayer nanodisks. The results of our simulations show that: (i) A reasonably strong DD interaction is also able to induce a single magnetic vortex on a small nanodisk which is stable in the whole low temperature region; the out-of-plane components of all spins are negligible, so it is pointless and irrelevant to talk about the chirality of this sort of vortex. (ii) A strong DM interaction usually generates a multi-vortex structure which evolves with varying temperature; the calculated average distance between two neighboring vortices agrees well with a grid theory at temperatures well below $T_{M}$; and since $D>0$, the chirality of every vortex is still right-handed despite the fact that the vortex has been considerably deformed. (iii) When a weak DD interaction is further considered, the multi-domains induced by the strong DM interaction merge to form a single stable vortex below $T_{M}$. (iv) If only the Heisenberg and DM interactions are considered, the calculated transition temperature is simply proportional to $S(S+1)$; and if the temperature is scaled with $T_{M}$, the magnetizations are divided by $S$, their curves only differ slightly from those obtained with different spin values in the whole magnetic phase.

To solve the Hamiltonians of many-spin systems, mean-field theory (MFT) has been implemented in our computational program. It is well known that the main idea of MFT is to replace all interactions to a spin with an effective magnetic field. This reduces a many-body problem to an effective one-body problem. However, in quantum field theory, the Hamiltonian may be expanded in terms of fluctuation$\mathrm{s}$ around the averaged field. This means the MFT does not consider fluctuations. MFT works better for high-dimensional systems. This can be explained by the weaker fluctuations because each spin is surrounded by more neighbors. The fluctuations are generally smaller when the system is away from the critical point. Therefore, it is expected that MFT will give more accurate results for such a situation. Especially, when long-range interactions are considered, more spins are coupled. This would lead to a strong dampening of the fluctuations with the result that MFT would become both qualitatively and quantitatively accurate.

For the nanodisks studied here, a spin is firstly considered to be coupled with its four nearest neighboring spins via both Heisenberg exchange and DM interactions, so a spin seems to interact with eight neighbors. Afterwards, the long-range DD interaction is taken into account, the effects of spin fluctuations are then greatly suppressed. Hence, we expect that MFT can provide rather accurate results in the 
both cases. Anyway, spin fluctuation is still able to reduce, though weakly, both transition temperature $T_{M}$ and magnetization in a narrow region below $T_{M}$. In that region, the orientation of each spin may be turned away slightly and randomly by the fluctuations from the direction shown in the above figures. In order to quantitatively investigate these effects, one must include such fluctuation terms in the model and carry out more simulations. This task is actually not very difficult.

As explained above, when only the DM and Heisenberg interactions co-exist, a quantity named DM length has been introduced and defined as $\zeta=\mathcal{J} / D$, in order to describe the self-organized multi-domain structures, where the distance between two nearest grids is defined as the unity [30]. However, here $\mathcal{J}$ and $D$ are the Heisenberg exchange and DM energy per grid, respectively, and each grid in the simulation represents $n \times n$ atomic sites. Thus $\zeta$ represents the averaged distance between two neighboring magnetic domains $[28,29,30]$. Moreover, we found recently that under a strong DM interaction, magnetic strips are formed on nanodisks. However, if a reasonably strong external magnetic field is applied in the perpendicular direction, the strips disappear and they are replaced by evenly distributed vortices.

Rocha et al. have performed Monte Carlo simulations to systematically investigate the conditions for the formation of vortices in nanodisks when Heisenberg and DD interactions are both present [41]. They found that, in nanodisk with triangular, square, or hexagonal structure, there is always a "transition" line separating the vortex state from a capacitor-like state. This line has a finite size scaling form that depends on the size, $L=2 R$, of the system as $d_{c}=d_{0}+1 /\left[A\left(1+B L^{2}\right)\right]$, where $d_{0}, A$ and $B$ are constants for each type of the lattice. This rule is obeyed by all three kinds of lattices. In the vortex phase there exist two sub-types of vortices: an in-plane and an out-of-plane vortex, which are separated by a constant line, $d=d_{c}$. They simulated nanodisks of several diameters $10 \leq L \leq 90$, but only displayed their calculated spin structure obtained with $d=0.1$ and $L=30$.

We found recently that in the presence of Heisenberg exchange, a strong DD interaction can also generate multi-vortex structure on large monolayer nanodisks. For instance, when $\mathcal{J}=1 \mathrm{~K}, d=0.5 \mathrm{~K}$ and $R=30 a$, an anti-vortex appears in the central region, it is surrounded by four vortices, every neighboring pair of the vortices curl in the opposite direction, and the whole pattern shows excellent symmetry. By increasing the strength of DD interaction, more vortices are observed. To check these results, we have just done simulations with our another quantum method, and found that the results obtained with the two approaches are all well consistent with each other. These results have been summarized in a separated paper.

When both DM and DD interactions are considered in simulations, the calculated vortical structures in general depend on the size of the disk and the strengths of the 
two intrinsic interactions. Moreover, the external magnetic field and the magnetic anisotropy also play important roles. Since both DM and DD interactions are very complicated, there is no a general rule to describe the vortical structures induced by both of them. However, for all these systems we can always investigate them numerically before such a rule is found.

At this juncture, we would like to stress out that all our results obtained so far with the quantum simulation approach are reasonable [24, 25, 26, 27, 28, 29]. In particular, our calculated magnetic structure and transition temperature for a $\mathrm{DyNi}_{2} \mathrm{~B}_{2} \mathrm{C}$ nanoball, assumed being cut out of a body-centered tetragonal crystalline, are in good agreement with those of the bulk sample [25]. So we believe that the results presented in this paper are reliable, the new phenomena observed and mechanisms revealed here might arouse the interest of both experimentalists and theoreticians in the field.

\section{Acknowledgements}

Z.-S. Liu acknowledges the financial support provided by National Natural Science Foundation of China under grant No. 11274177. O. Ciftja was supported in part by U.S. Army Research Office (ARO) Grant No. W911NF-13-1-0139 and National Science Foundation (NSF) Grant No. DMR-1410350. And H. Ian is supported by the FDCT of Macau under grant 013/2013/A1, University of Macau under grants MRG022 /IH/2013/FST and MYRG2014-00052-FST, and National Natural Science Foundation of China under Grant No.11404415.

\section{References}

[1] A. N. Bogdanov, and D. A. Yablonskii, Sov. Phys. JETP 68 (1989) 101.

[2] T. H. R. Skyrme, Nucl. Phys. 31 (1962) 556.

[3] I. E. Dzyaloshinsky, J. Phys. Chem. Solids 4 (1958) 241.

[4] T. Moriya, Phys. Rev. Lett. 4 (1960) 228.

[5] T. Moriya, Phys. Rev. 120 (1960) 91.

[6] W. Münzer, A. Neubauer, T. Adams, S. Mühlbauer, C. Franz, F. Jonietz, R. Georgii, P. Böni, B. Pedersen, M. Schmidt, A. Rosch, and C. Pfleiderer, Phys. Rev. B 81 (2010) 041203. 
[7] X. Yu, Y. Onose, N. Kanazawa, J. H. Park, J. H. Han, Y. Matsui, N. Nagaosa, and Y. Tokura, Nature 465 (2010) 901.

[8] A. Tonomura, X. Yu, K. Yanagisawa, T. Matsuda, Y. Onose, N. Kanazawa, H. S. Park, and Y. Tokura, Nano Lett. 12 (2012) 1673.

[9] S. Mühlbauer, B. Binz, F. Jonietz, C. Pfleiderer, A. Rosch, A. Neubauer, R. Georgii, and P. Böni, Science 323 (2009) 915.

[10] S. Heinze, K. von Bergmann, M. Menzel, J. Brede, A. Kubetzka, R. Wiesendanger, G. Bihlmayer, and S. Blügel, Nat. Phys. 7 (2011) 713.

[11] T. Adams, S. Mühlbauer, A. Neubauer, W. Münzer, F. Jonietz, R. Georgii, B. Pedersen, P. Böni, A. Rosch, and C. Pfleiderer, J. Phys.: Conf. Ser. 200 (2010) 032001.

[12] T. Adams, S. Mühlbauer, C. Pfleiderer, F. Jonietz, A. Bauer, A. Neubauer, R. Georgii, P. Böni, U. Keiderling, K. Everschor, M. Garst, and A. Rosch, Phys. Rev. Lett. 107 (2011) 217206.

[13] A. N. Bogdanov, and U. K. Rößler, Phys. Rev. Lett. 87 (2001) 037203.

[14] A. Bogdanov, and A. Hubert, J. Magn. Magn. Mater. 138 (1994) 255.

[15] C. Pfleiderer, and A. Rosch, Nature 465 (2010) 880.

[16] S.-H. Chung, R. D. McMichael, D. T. Pierce, and J. Unguris, Phys. Rev. B 81 (2010) 024410.

[17] N. S. Kiselev, A. N. Bogdanov, R. Schäfer, and U. K. Rößler, J. Phys. D: Appl. Phys. 44 (2011) 392001.

[18] F. Jonietz, S. Mühlbauer, C. Pfleiderer, A. Neubauer, W. Münzer, A. Bauer, T. Adams, R. Georgii, P. Böni, R. A. Duine, K. Everschor, M. Garst, and A. Rosch, Science 330 (2010) 1648.

[19] X. Z. Yu, N. Kanazawa, W. Z. Zhang, T. Nagai, T. Hara, K. Kimoto, Y. Matsui, Y. Onose, and Y. Tokura, Nat. Commun. 3 (2012) 988.

[20] L.Thomas, R. Moriya, C. Rettner, and S. S. P. Parkin, Science 330 (2010) 1810.

[21] A. Fert, V. Cros, and J. Sampaio, Nat. Nanotechnol. 8 (2013) 152. 
[22] S. S. P. Parkin, M. Hayashi, and L. Thomas, Science 320 (2008) 190.

[23] X. Zhang, G. P. Zhao, H. Fangohr, J. P. Liu, and W. X. Xia, Sci. Rep. 5 (2015) 1.

[24] Z.-S. Liu, V. Sechovský, and M. Diviš, J. Phys.: Condens. Matter 23 (2011) 016002 .

[25] Z.-S. Liu, V. Sechovský, and M. Diviš, Phys. Status Solidi B 249 (2012) 202.

[26] Z.-S. Liu, V. Sechovský, and M. Diviš, Physica E 44 (2012) 826.

[27] Z.-S. Liu, V. Sechovský, and M. Diviš, Physica E 62 (2014) 123.

[28] Z.-S. Liu, and H. Ian, J Nanopart. Res. 18 (2016) 9.

[29] Z.-S. Liu, and H. Ian, Chem. Phys. Lett. 649 (2016) 135.

[30] H. Y. Kwon, K. M. Bu, Y. Z. Wu, and C. Won, J. Magn. Magn. Mater. 324 (2012) 2171.

[31] B. H. Kim, and B. I. Min, New J. Phys. 13 (2011) 073034.

[32] S. Prakash, Phys. Rev. B 42 (1990) 6574.

[33] A. B. MacIsaac, J. P. Whitehead, K. De'Bell, and P. H. Poole, Phys. Rev. Lett. 77 (1996) 739.

[34] A. Hucht, and K. D. Usadel, J. Magn. Magn. Mater. 156 (1996) 423.

[35] A. B. MacIsaac, K. De'Bell, and J. P. Whitehead, Phys. Rev. Lett. 80 (1998) 616.

[36] E. Yu. Vedmedenko, A. Ghazali, and J. -C. S. Lévy, Phys. Rev. B 59 (1999) 3329.

[37] E. Yu. Vedmedenko, H. P. Oepen, A. Ghazali, J.-C. S. Lévy, and J. Kirschner, Phys. Rev. Lett. 84 (2000) 5884.

[38] V. E. Kireev, and B. A. Ivanov, Phys. Rev. B 68 (2003) 104428.

[39] S. A. Leonel, I. A. Marques, P. Z. Coura, and B. V. Costa, J. Appl. Phys. 102 (2007) 104311. 
[40] L. Berger, Y. Labaye, M. Tamine, and J. M. D. Coey, Phys. Rev. B 77 (2008) 104431.

[41] J. C. S. Rocha, P. Z. Coura, S. A. Leonel, R. A. Dias, and B. V. Costa1, J. Appl. Phys. 107 (2010) 053903.

[42] I. Booth, A. B. Isaac, J. P. Whitehead, and K. De'Bell, Phys. Rev. Lett. 75 (1995) 951.

[43] J. Sasaki, and F. Matsubara, J. Phys. Soc. Japan 66 (1997) 2138.

[44] E. Y. Vedmedenko, A. Ghazali, and J.-C. S. Lévy, Surf. Sci. 402 (1998) 391.

[45] R. P. Cowburn, and M. E. Welland, Phys. Rev. B 58 (1998) 9217.

R. P. Cowburn, D. K. Koltsov, A. O. Adeyeye, M. E. Welland, and D.M. Tricker, Phys. Rev. Lett. 83 (1999) 1042.

[46] B. A. Ivanov, H. J. Schnitzer, F. G. Mertens, and G. M. Wysin, Phys. Rev. B 58 (1998) 8464.

[47] J.-C. S. Lévy, Phys. Rev. B 63 (2001) 104409.

[48] A. Vaterlaus, C. Stamm, U. Maier, M. G. Pini, P. Politi, and D. Pescia, Phys. Rev. Lett. 84 ( 2000) 224.

[49] J. Li, and C. Rau, Phys. Rev. Lett. 97 (2006) 107201.

[50] J. E. Villegas, C. P. Li, and I. K. Schuller, Phys. Rev. Lett. 99 (2007) 227001.

[51] M. Gong, Y. Y. Qian, M. Yan, V.W. Scarola, and C. W. Zhang, Sci. Rep. 5 (2015) 10050.

[52] Y. M. Luo, C. Zhou, C. Won, and Y. Z. Wu, AIP Adv. 4 (2014) 047136.

[53] T. Shinjo, T. Okuno, R. Hassdorf, K. Shigeto, and T. Ono, Science 289 (2000) 930.

[54] A. Wachowiak, J. Wiebe, M. Bode, O. Pietzsch, M. Morgenstern, and R. Wiesendanger, Science 298 (2002) 577.

[55] S. B. Choe, Y. Acremann, A. Scholl, A. Bauer, A. Doran, J. Stöhr, and H. A. Padmore, Science 304 (2004) 420. 
[56] M. Y. Im, P. Fischer, K. Yamada, T. Sato, S. Kasai, Y. Nakatani, T. Ono, Nat. Commun. 3 (2012) 983. 

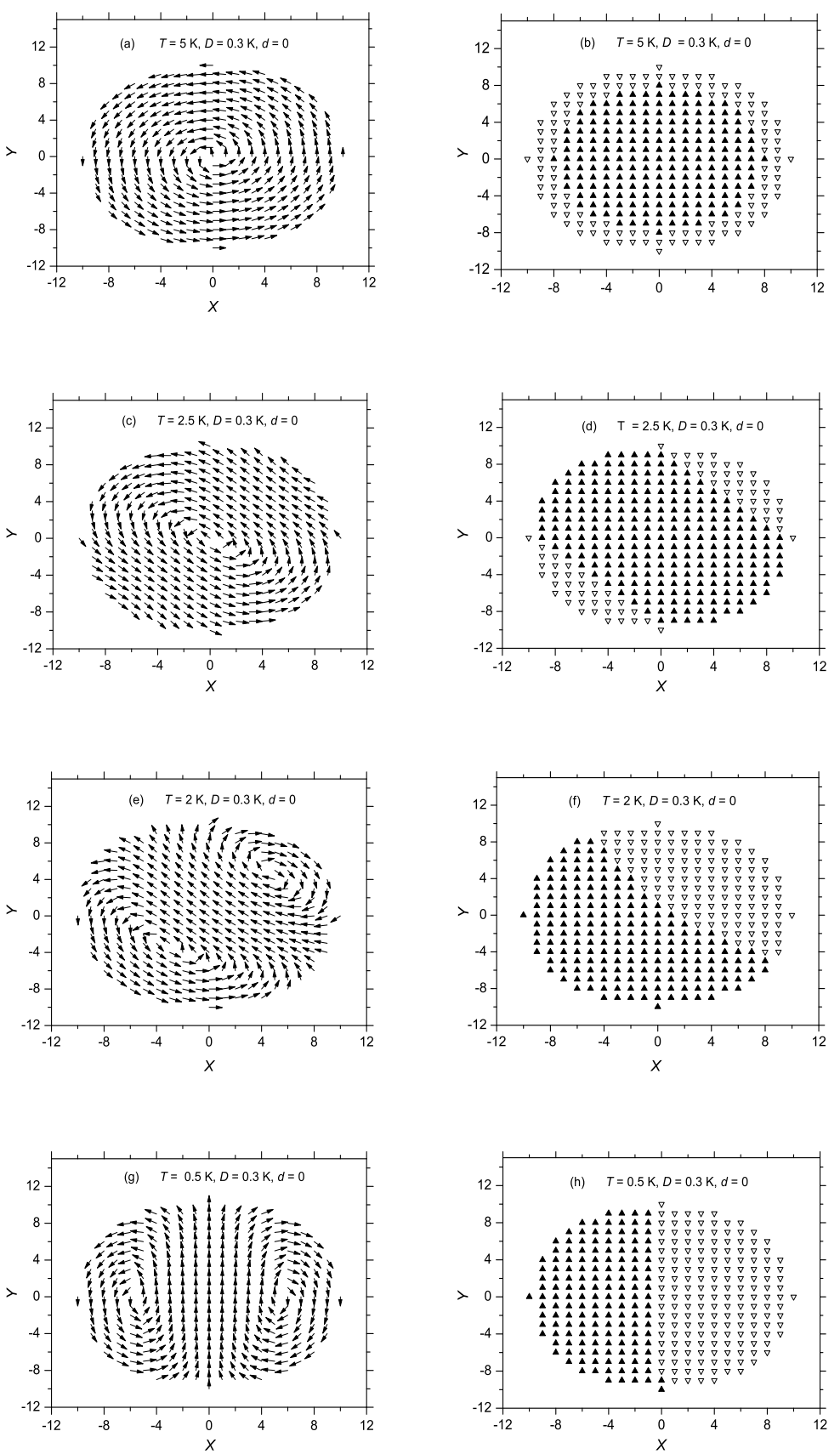

Figure 2. Calculated spin configurations projected onto: (a,c,e,g) the disk plane, and $(\mathrm{b}, \mathrm{d}, \mathrm{f}, \mathrm{h})$ the normal $z$-direction, at four temperatures. Here $R=$ $10 \mathrm{a}, \mathcal{J}=1 \mathrm{~K}, D=0.3 \mathrm{~K}$, and $d=0$, respectively. 

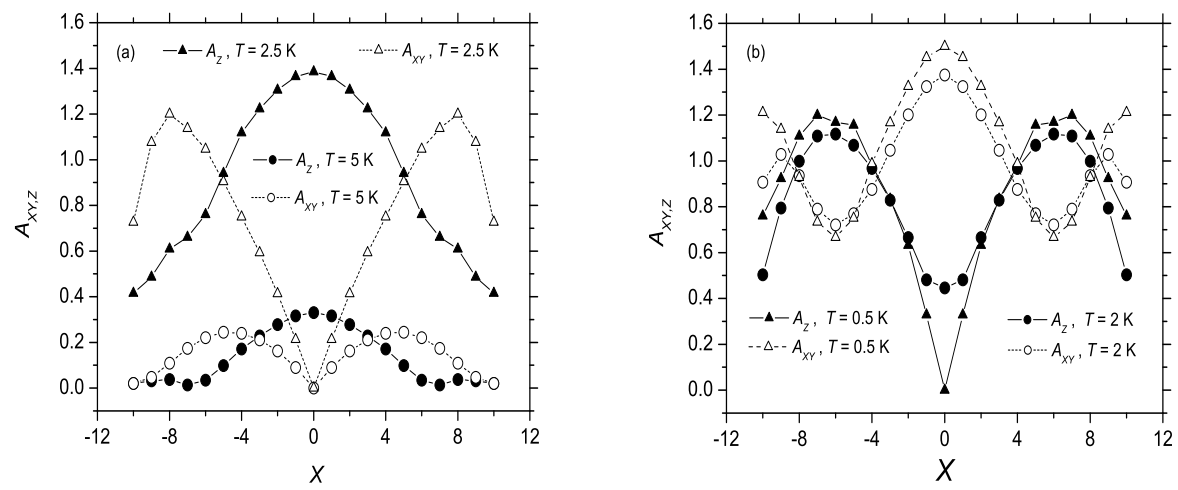

Figure 3. Calculated $A_{z}(r)$ and $A_{x y}(r)$ as functions of the distances from the center of the nanodisk at (a) $T=5 \mathrm{~K}, 2.5 \mathrm{~K}$, and (b) $T=2 \mathrm{~K}, 0.5 \mathrm{~K}$, respectively. Here $R=10 \mathrm{a}, \mathcal{J}=1 \mathrm{~K}, D=0.3 \mathrm{~K}$, and $d=0$, respectively.
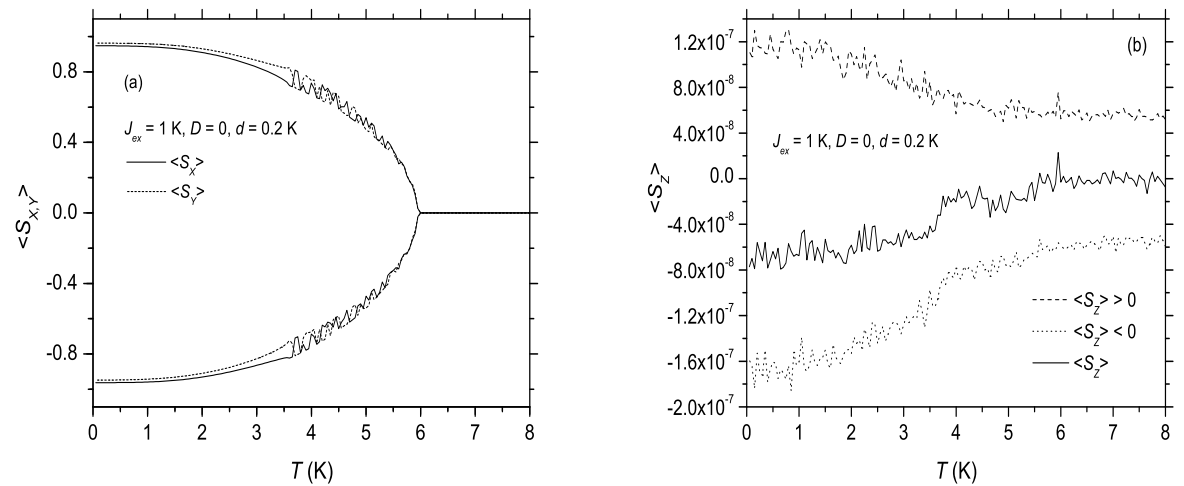

Figure 4. Calculated spontaneous (a) $\left\langle S_{x}\right\rangle,\left\langle S_{y}\right\rangle$ and (b) $\left\langle S_{z}\right\rangle$ for the monolayer FM nanodisk as functions of temperature. Here $R=10 \mathrm{a}, \mathcal{J}=1 \mathrm{~K}, D$ $=0 \mathrm{~K}$, and $d=0.2 \mathrm{~K}$, respectively. 

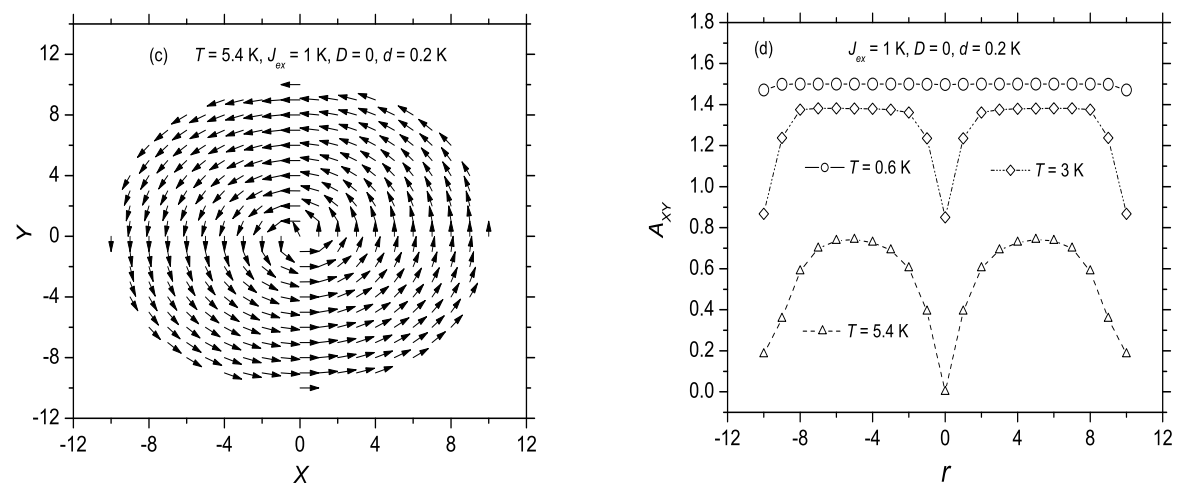

Figure 5. Calculated (a) spin configuration projected onto the disk plane, and (b) $A_{x y}$ as functions of the distances from the disk center at three different temperatures. Here $R=10 \mathrm{a}, \mathcal{J}=1 \mathrm{~K}, D=0 \mathrm{~K}$, and $d=0.2$, respectively.
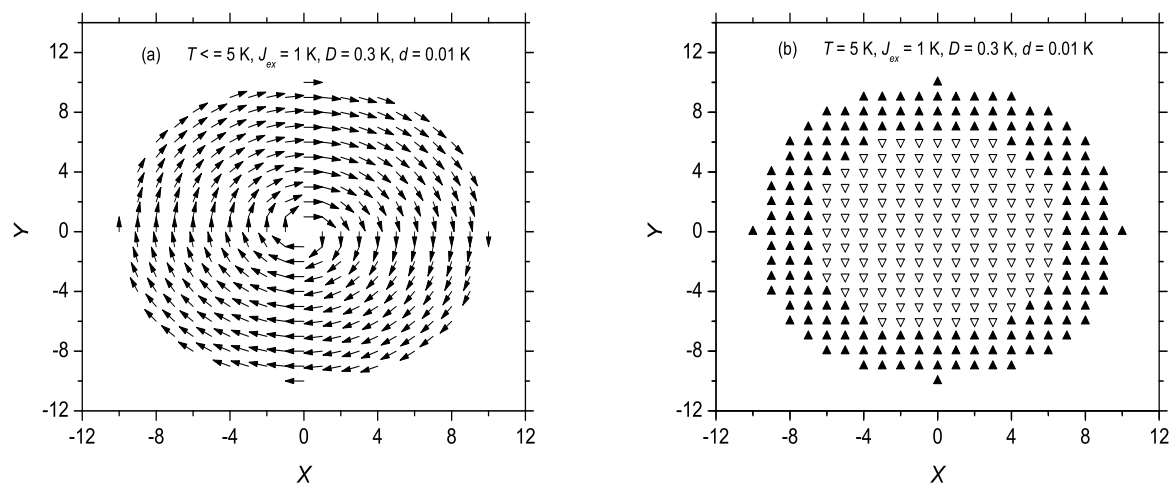

Figure 6. Calculated spin configuration projected onto the (a) $x y$-plane, and (b) $z$-direction in the magnetic phase. Here $R=10 \mathrm{a}, \mathcal{J}=1 \mathrm{~K}, D=0.3 \mathrm{~K}$, and $d=0.01 \mathrm{~K}$, respectively. 

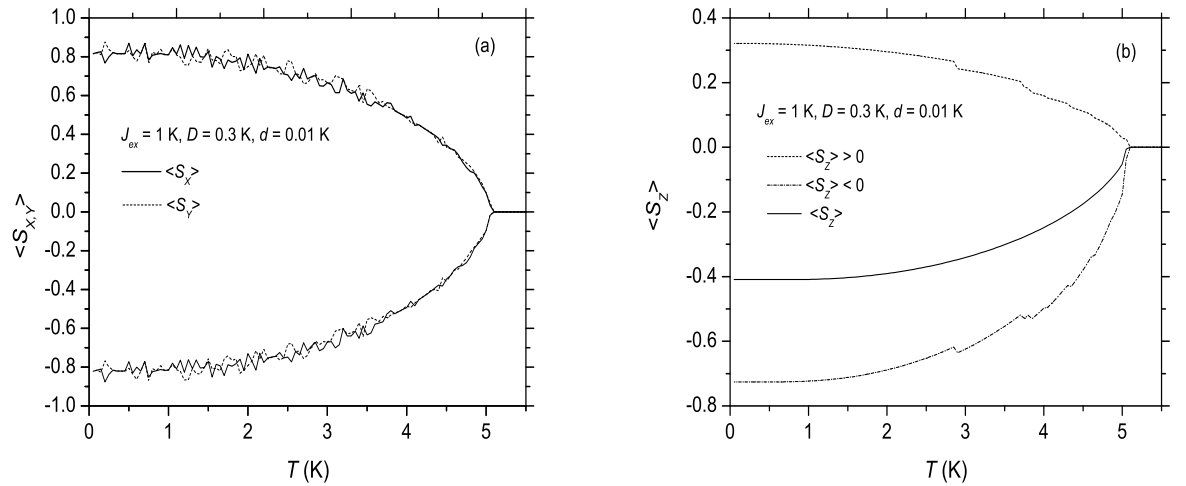

Figure 7. Calculated spontaneous (a) $\left\langle S_{x}\right\rangle,\left\langle S_{y}\right\rangle$, and (b) $\left\langle S_{z}\right\rangle$ for the monolayer nanodisk as the functions of temperature. Here $R=10 \mathrm{a}, \mathcal{J}=1 \mathrm{~K}, D$ $=0.3 \mathrm{~K}$, and $d=0.01 \mathrm{~K}$, respectively. 